\title{
Spectroscopic observations of young open clusters: IC 1805, NGC 654 and NGC 6823
}

\author{
H.M. Shi ${ }^{1,2}$ and J.Y. Hu ${ }^{2,3}$ \\ 1 Shanghai Astronomical observatory, Chinese Academy of Sciences, Shanghai 200030, China \\ 2 Joint Laboratories for Optical Astronomy, Chinese Academy of Sciences, China \\ 3 Beijing Astronomical observatory, Chinese Academy of Sciences, Beijing, 100080, China
}

Received September 30, 1998; accepted January 11, 1999

\begin{abstract}
Spectroscopic observations were made for a sample of 263 stars from three young open clusters IC 1805, NGC 654 and NGC 6823 with CCD detectors in the blue and yellow-red spectral regions. MKK types were properly determined for these stars by carefully comparing spectra of program stars with those of a complete grid of MKK standards, which were observed with the same instruments as the program stars. For most of the observed stars precise MKK types were assigned for the first time. A few of early-type emission-line stars were discovered or confirmed. The presence of Be stars in the studied clusters is discussed. Spectra of the observed MKK standards also reveal lots of features showing temperature or luminosity effect in the yellow-red region, which helped in our stellar spectral classification.
\end{abstract}

Key words: stars: fundamental parameters - stars: emission-line, $\mathrm{Be}$ - open clusters and associations: IC 1805, NGC 654 and NGC 6823

\section{Introduction}

Open clusters are natural laboratories for stellar evolution, because stars in clusters share a common origin from the same progenitor molecular cloud. Young open clusters are particularly suited to study the processes of star formation and early evolution of stars over a wide mass spectrum. Many questions related to young clusters and/or associations remain to be answered, e.g., the constancy of IMF (at higher masses), the coevality of star formation, the evolution of massive stars and its influence on the environment, and the presence of various peculiar stars (emission-line stars, for example) and their evolutionary

Send offprint requests to: H.M. Shi

e-mail: hmshi@center.shao.ac.cn
Table 1. Spectrographs and gratings

\begin{tabular}{|c|c|c|c|c|}
\hline \multirow{2}{*}{$\begin{array}{c}\text { spectrograph } \\
\text { name }\end{array}$} & \multirow{2}{*}{$\begin{array}{l}\text { grating } \\
\text { lines } / \mathrm{mm}\end{array}$} & \multicolumn{2}{|c|}{ dispersion } & \multirow{2}{*}{$\begin{array}{c}\text { central wavelenth } \\
\AA\end{array}$} \\
\hline & & $\AA / \mathrm{mm}$ & $\AA /$ pixel & \\
\hline UNIVERSAL & 300 & 195 & 4.7 & 6000 \\
\hline \multirow[t]{3}{*}{ OMR } & 300 & 200 & 4.8 & 6000 \\
\hline & 600 & 100 & 2.4 & 5000 \\
\hline & 1200 & 50 & 1.2 & 4375 \\
\hline
\end{tabular}

status, to just cite a few. The study of clusters and associations deeply relies upon the accumulation of various kinds of precise data, be it astrometric, photometric or spectroscopic. While large efforts have been spent on obtaining magnitudes and colours in various systems such as $U B V$ and uvby for stars in clusters, the situation about spectroscopic data is still far from satisfactory. Reliable MKK spectral types, for example, are often available in published literature only to few brightest stars of a cluster (Mermilliod 1986).

Accurate two-dimensional spectral classification (temperature type and luminosity class) of stars is essential to the study of young open clusters. Differential reddenings often affect member stars in young clusters, causing scatters in color-magnitude diagrams. Spectroscopy provides the means of precisely detecting any differential reddening. By combination with photometric data (magnitudes and colors), spectral classification may be used to construct individually-dereddened high-quality $\mathrm{H}-\mathrm{R}$ diagrams, from which the distances and evolutionary ages of clusters can be reliably determined and even the effects of binarity (usually indistinguishable from small diffential reddenings) may be examined. Spectral classification can also be used to investigate the nature of reddening and extinction, determining if any abnormality exists and if the differential reddening is caused by interstellar, intracluster or circumstellar extinction material (Sagar 1987; van den Ancker et al. 1997). Peculiar stars (e.g., emission-line stars) may also be discovered from spectral classification. 
Traditionally spectral classification is made photographically in the blue spectral region of $3900 \AA$ through $4900 \AA$. Reliable two-dimensional spectral types can be gained only for brighter stars due to the low sensitivity of photographic plates. With the use of CCDs spectroscopy can be done on much fainter objects and on a broader spectral coverage. The peak of the quantum efficiency of CCDs is usually in the yellow-red spectral region, which is advantageous to the observation of late type stars or highly-reddened early type stars such as those in young open clusters. With the spectral coverage extended from the blue into the yellow-red and even the near infrared, additional spectral features suitable for stellar classification also appear in these regions of longer wavelength (Jacoby et al. 1984; Turnshek et al. 1985; Torres-Dodgen \& Weaver 1993; Danks \& Dennefeld 1994; Shi \& Hu 1999).

We have begun a project of systematically investigating the properties and stellar contents of young open clusters and/or associations, especially those with severe differential reddening. The study is to be based upon more complete and homogeneous sets of combined data. As a part of this program and the first step, we engage ourselves in making accurate two-dimensional spectral classification for large samples of stars in young clusters. The first three clusters selected by us are IC $1805(\mathrm{C} 0228+612$, $\left.\alpha_{1950}=02^{\mathrm{h}} 28^{\mathrm{m}} \cdot 9, \delta_{1950}=61^{\circ} 54^{\prime}\right), \mathrm{NGC} 654(\mathrm{C} 0140+616$, $\left.\alpha_{1950}=01^{\mathrm{h}} 40^{\mathrm{m}} 6, \quad \delta_{1950}=61^{\circ} 38^{\prime}\right)$ and NGC 6823 $\left(\mathrm{C} 1941+231, \alpha_{1950}=19^{\mathrm{h}} 41^{\mathrm{m}} 0, \quad \delta_{1950}=23^{\circ} 11^{\prime}\right)$. They are of very young ages and all showed differential reddenings according to earlier investigators (Moffat 1972; Turner 1979; Stone 1980; Sagar \& Joshi 1981; Joshi \& Sagar 1983a,b). Most of the cluster stars lack MKK types. In this work a total of 263 stars from these three clusters were spectroscopically observed by slit spectrograh and CCD detectors in the blue and yellow-red spectral regions. Results of the MKK classification are given in this paper. The presence of emission-line stars is discussed. Further studies upon these clusters are underway.

\section{Observation and data reduction}

Spectroscopic data were obtained during two observing runs, in 1995 and in 1997, with the $2.16 \mathrm{~m}$ telescope at Beijing Astronomical Observatory. In 1995 the telescope was equipped with a Tektronix 1024 CCD mounted at an UNIVASAL spectrograph, whereas in 1997 with another Tektronix 1024 CCD at an OMR (Optomechanics Research Inc.) spectrograph. The pixel size of the two Tektronix CCDs were both $24 \mu \mathrm{m}$. For the UNIVASAL spectrograph a grating of 300 lines/mm were used, and for the OMR spectrograph three gratings of 300 lines $/ \mathrm{mm}$, 600 lines/mm and 1200 lines/mm were used. The corresponding dispersion and central wavelength are given in Table 1. A slit with a width of about $2^{\prime \prime}$ was used during all the observations.
A total of 263 program stars, 140 from IC 1805, 46 from NGC 654 and 77 from NGC 6823, were observed. They are mainly proper motion cluster members (See Sect. 3.2 ). The magnitude limit is around $V=14^{\mathrm{m}}$. Spectra were taken with the 300 lines/mm grating for nearly all program stars. For the brighter stars spectra were also taken with the 600 lines $/ \mathrm{mm}$ grating, and for the few brightest stars additional spectra were obtained with the 1200 lines/mm grating. The exposure times ranged from a few minutes to about an hour, depending on the magnitude of the object, the seeing, and the grating used. The resulting signal-to-noise $(\mathrm{S} / \mathrm{N})$ ratios usually exceeded 100. The observed cluster stars with their identifying numbers are listed in Tables 2, 3, and 4 for IC 1805, NGC 654 and NGC 6823 respectively.

We also observed a complete grid of MKK standards (up to 200) with the same instruments (telescope and spectrograph with same gratings) and under nearly identical observing conditions as our program stars. The $\mathrm{S} / \mathrm{N}$ was kept comparable to that of the program stars. These standard stars are mainly from Keenan \& McNeil (1976); Morgan et al. (1978) and Morgan \& Keenan (1973). Reobserving MKK standards here is necessary for the spectral comparison and classification.

All the spectra were reduced using MIDAS. The raw CCD spectral images were corrected using average Bias and high $\mathrm{S} / \mathrm{N}$ dome-flat frames. The dark current of the used CCDs was negligible. Sky subtraction was achieved by subtracting a third degree polynomial fitted to the spectrum in the spatial direction. This kind of sky subtraction proved to be effective in eliminating the emission from surrounding nebulosity. The sky-subtracted spectral images were then wavelength-calibrated using the comparison spectra (Fe-Ar or He-Ne-Ar lamps) and onedimensional spectra were extracted. For the purpose of spectral classification all one-dimensional spectra were continuum-normalized instead of flux-calibrated. Data reduction for program stars and MKK standard stars were the same. Representative spectra of MKK standards are illustrated in Fig. ?? and Fig. ??. Spectra of all the program stars from the three clusters are shown in Fig. ?? through Fig. ??.

\section{Spectral classification}

\subsection{Methods}

To properly classify stellar spectra in the MKK system, the spectra of program stars have to be carefully compared with those of MKK standards. MKK standards should be observed with the same instrument and under the same conditions as the program stars. The reobservation of MKK standards is necessary to link the local instrumental system to the MKK system which is defined by its standards (Morgan \& Keenan 1973; Morgan 1984). 
Table 2. Spectral classification of stars in IC 1805

\begin{tabular}{|c|c|c|c|c|c|c|}
\hline $\begin{array}{l}\text { star } \\
\text { No. }\end{array}$ & $\begin{array}{c}p \\
(\%)\end{array}$ & $\begin{array}{l}\text { spectral-type } \\
\text { published }\end{array}$ & sources & $\begin{array}{l}\text { spectral-type } \\
\text { new }\end{array}$ & $\begin{array}{l}\text { grating } \\
\text { No. }\end{array}$ & $\overline{\text { notes }}$ \\
\hline 5 & 53 & & & G8II-III & 1 & \\
\hline 8 & 75 & & & $\mathrm{G} 2 \mathrm{~V}$ & 1 & \\
\hline 18 & 78 & & & $\mathrm{~B} 1 \mathrm{~V}$ & 1 & \\
\hline 21 & 75 & O9.5V, B0III & $a, b$ & B0III & 1,2 & \\
\hline 23 & 83 & $\mathrm{~B} 1 \mathrm{~V}$ & $\mathrm{~b}$ & $\mathrm{~B} 1 \mathrm{~V}$ & 1 & \\
\hline 26 & 62 & & & A3II-III & 1 & \\
\hline 29 & 8 & & & $\mathrm{~A} 7 \mathrm{~V}$ & 1 & \\
\hline 39 & 84 & & & $\mathrm{~A} 3 \mathrm{~V}$ & 1 & \\
\hline 42 & 62 & $\mathrm{~B} 2.5 \mathrm{~V}$ & $\mathrm{a}$ & $\mathrm{B} 2.5 \mathrm{~V}$ & 1 & \\
\hline 43 & 78 & & & $\mathrm{G} 2 \mathrm{~V}$ & 1 & \\
\hline 49 & 72 & $\mathrm{~B} 2.5 \mathrm{~V}$ & $\mathrm{a}$ & $\mathrm{B} 2 \mathrm{~V}$ & 1 & \\
\hline 50 & 80 & & & F3V & 1 & \\
\hline 52 & 73 & $\mathrm{~B} 2.5 \mathrm{~V}$ & $\mathrm{a}$ & $\mathrm{B} 2 \mathrm{~V}$ & 1 & \\
\hline 53 & 70 & & & $\mathrm{~B} 1.5 \mathrm{~V}$ & 1 & \\
\hline 55 & 83 & & & F0III & 1 & \\
\hline 56 & 35 & & & $\mathrm{~A} 0 \mathrm{~V}$ & 1 & \\
\hline 57 & 75 & & & F3III & 1 & \\
\hline 62 & 87 & & & $\mathrm{~B} 2.5 \mathrm{~V}$ & 1 & \\
\hline 63 & 80 & & & F1V & 1 & \\
\hline 68 & 82 & & & $\mathrm{~B} 2.5 \mathrm{~V}$ & 1 & \\
\hline 69 & 82 & $\mathrm{~B} 2.5 \mathrm{~V}$ & $\mathrm{a}$ & $\mathrm{B} 2 \mathrm{~V}$ & 1 & \\
\hline 70 & 85 & B0.5Ia & $\mathrm{b}$ & B0.5Iae & 1,2 & \\
\hline 71 & 52 & & & A3II & 1 & \\
\hline 72 & 80 & $\mathrm{~B} 2.5 \mathrm{~V}$ & $\mathrm{a}$ & $\mathrm{B} 1 \mathrm{~V}$ & 1 & \\
\hline 74 & 63 & K0II & $\mathrm{b}$ & G8II & 1,2 & \\
\hline 82 & 84 & $\mathrm{~B} 2.5 \mathrm{~V}$ & $\mathrm{a}$ & $\mathrm{B} 1.5 \mathrm{~V}$ & 1 & \\
\hline 85 & 73 & & & $\mathrm{~B} 5 \mathrm{~V}$ & 1 & \\
\hline 91 & 78 & & & A3III & 1 & \\
\hline 94 & 62 & $\mathrm{~B} 2 \mathrm{~V}$ & a & $\mathrm{B} 2.5 \mathrm{~V}$ & 1 & \\
\hline 96 & 85 & & & $\mathrm{~B} 4 \mathrm{~V}$ & 1 & \\
\hline 99 & 86 & & & $\mathrm{~A} 6 \mathrm{~V}$ & 1 & \\
\hline 103 & 84 & $\mathrm{~B} 1 \mathrm{~V}, \mathrm{~B} 0 \mathrm{~V}$ & $\mathrm{a}, \mathrm{c}$ & $\mathrm{B} 1 \mathrm{~V}$ & 1,2 & \\
\hline 104 & 79 & $\mathrm{O} 7 \mathrm{~V}((\mathrm{f}))$ & $\mathrm{a}$ & $\mathrm{O} 7 \mathrm{~V}$ & 1,2 & \\
\hline 105 & 76 & A1II-III & $\mathrm{b}$ & A7III & 1 & \\
\hline 110 & 60 & & & A8III & 1 & \\
\hline 111 & 87 & $\mathrm{~B} 1 \mathrm{~V}, \mathrm{~B} 2 \mathrm{~V}$ & $\mathrm{a}$, & $\mathrm{B} 1 \mathrm{~V}$ & 1,2 & \\
\hline 112 & 84 & $\mathrm{~B} 0.2 \mathrm{~V}, \mathrm{~B} 0 \mathrm{~V}, 09.5 \mathrm{~V}$ & $a, b, d$ & $\mathrm{~B} 0 \mathrm{~V}$ & $1,2,3$ & \\
\hline 113 & 85 & $\mathrm{Be}, \mathrm{O} 9 \mathrm{Ve}$ & $\mathrm{a}, \mathrm{b}$ & O9.5Ve & $1,2,3$ & $\mathrm{~A}$ \\
\hline 118 & 86 & $09.5 \mathrm{~V}((\mathrm{f})), \mathrm{O} 9 \mathrm{~V}$ & $\mathrm{a}, \mathrm{b}$ & $\mathrm{O} 9 \mathrm{~V}$ & $1,2,3$ & \\
\hline 121 & 74 & $\mathrm{~B} 1 \mathrm{~V}$ & $\mathrm{a}$ & $\mathrm{B} 1.5 \mathrm{~V}$ & 1,2 & \\
\hline 122 & 71 & & & F8V & 1 & \\
\hline 123 & 70 & & & $\mathrm{~B} 9.5 \mathrm{~V}$ & 1 & \\
\hline 124 & 75 & & & $\mathrm{~A} 5 \mathrm{~V}$ & 1 & \\
\hline 128 & 83 & & & $\mathrm{~A} 8 \mathrm{~V}$ & 1 & \\
\hline 129 & 83 & & & $\mathrm{~B} 5 \mathrm{~V}$ & 1 & \\
\hline 130 & 40 & & & B3V & 1 & \\
\hline 131 & 60 & & & G3V & 1 & \\
\hline 134 & 85 & & & $\mathrm{~B} 7 \mathrm{~V}$ & 1 & \\
\hline 136 & 77 & $\mathrm{~B} 1.5 \mathrm{~V}, \mathrm{~B} 1 \mathrm{~V}$ & $a, b$ & $\mathrm{~B} 1.5 \mathrm{~V}$ & 1,2 & \\
\hline 138 & 80 & $\mathrm{O} 7 \mathrm{~V}((\mathrm{f})), \mathrm{O} 6.5 \mathrm{~V}$ & $\mathrm{a}, \mathrm{d}$ & $\mathrm{O} 7 \mathrm{~V}$ & $1,2,3$ & \\
\hline 139 & 83 & & & $\mathrm{~B} 4 \mathrm{~V}$ & 1 & \\
\hline 143 & 86 & $\mathrm{~B} 1 \mathrm{~V}$ & $\mathrm{a}$ & $\mathrm{B} 0.5 \mathrm{~V}$ & 1,2 & \\
\hline 146 & 30 & & & $\mathrm{~B} 5 \mathrm{~V}$ & 1 & \\
\hline 147 & 75 & & & $\mathrm{~B} 6 \mathrm{~V}$ & 1 & \\
\hline 148 & 6 & O4III(f), O5f & $a, d$ & O5III(f) & $1,2,3$ & \\
\hline 149 & 86 & $\mathrm{~B} 1.5 \mathrm{~V}, \mathrm{~B} 3 \mathrm{~V}, \mathrm{~B} 3 \mathrm{~V}$ & $\mathrm{a}, \mathrm{b}, \mathrm{c}$ & B2.5IV & 1 & \\
\hline 152 & 77 & & & B3V & 1,2 & \\
\hline
\end{tabular}


Table 2. continued

\begin{tabular}{|c|c|c|c|c|c|c|}
\hline $\begin{array}{l}\text { star } \\
\text { No. }\end{array}$ & $\begin{array}{c}p \\
(\%)\end{array}$ & $\begin{array}{l}\text { spectral-type } \\
\text { published }\end{array}$ & sources & $\begin{array}{l}\text { spectral-type } \\
\text { new }\end{array}$ & $\begin{array}{l}\text { grating } \\
\text { No. }\end{array}$ & $\overline{\text { notes }}$ \\
\hline 154 & 76 & & & $\mathrm{G} 2 \mathrm{~V}$ & 1 & \\
\hline 155 & 40 & & & $\mathrm{~A} 0 \mathrm{~V}$ & 1 & \\
\hline 156 & 62 & B1IV & $\mathrm{a}$ & $\mathrm{B} 1 \mathrm{~V}$ & 1,2 & \\
\hline 157 & 77 & & & $\mathrm{~B} 2.5 \mathrm{~V}$ & 1 & \\
\hline 158 & 67 & & & $\mathrm{~B} 1.5 \mathrm{~V}$ & 1,2 & \\
\hline 159 & 39 & & & B3V & 1 & \\
\hline 160 & 64 & O4If, O5Iaf, O4f & $\mathrm{a}, \mathrm{b}, \mathrm{d}$ & O4.5If & 1 & $\mathrm{~A}$ \\
\hline 161 & 76 & $\mathrm{~B} 1 \mathrm{~V}, \mathrm{~B} 1.5 \mathrm{~V}$ & $a, b$ & $\mathrm{~B} 1.5 \mathrm{~V}$ & 1,2 & \\
\hline 162 & 57 & & & $\mathrm{~B} 2.5 \mathrm{~V}$ & 1 & \\
\hline 163 & 84 & $\mathrm{~B} 1.5 \mathrm{~V}$ & $\mathrm{a}$ & $\mathrm{B} 2 \mathrm{~V}$ & 1 & \\
\hline 164 & 66 & & & $\mathrm{~B} 5 \mathrm{~V}$ & 1 & \\
\hline 165 & 79 & & & B3V & 1 & \\
\hline 166 & 84 & $\mathrm{~B} 2.5 \mathrm{~V}, \mathrm{~B} 3 \mathrm{~V}$ & $a, b$ & $\mathrm{~B} 2 \mathrm{~V}$ & 1,2 & \\
\hline 167 & 86 & $\mathrm{~B} 1.5 \mathrm{~V}$ & $\mathrm{a}$ & $\mathrm{B} 1.5 \mathrm{~V}$ & 1 & \\
\hline 168 & 65 & & & $\mathrm{~B} 8 \mathrm{~V}$ & 1 & \\
\hline 169 & 82 & $\mathrm{~B} 1.5 \mathrm{~V}, \mathrm{~B} 2 \mathrm{IV}$ & $a, b$ & B2III & 1,2 & \\
\hline 170 & 75 & B2.5V, A2II, A2II & $\mathrm{a}, \mathrm{b}, \mathrm{e}$ & A2III & $1,2,3$ & \\
\hline 171 & 70 & & & $\mathrm{~B} 5 \mathrm{~V}$ & 1 & \\
\hline 172 & 82 & & & $\mathrm{~B} 7 \mathrm{~V}$ & 1 & \\
\hline 174 & 80 & $\mathrm{~B} 2.5 \mathrm{~V}, \mathrm{~B} 2 \mathrm{~V}$ & $\mathrm{a}, \mathrm{b}$ & $\mathrm{B} 2 \mathrm{~V}$ & 1,2 & \\
\hline 175 & 82 & & & $\mathrm{~B} 2.5 \mathrm{~V}$ & 1 & \\
\hline 177 & 78 & & & B5V & 1 & \\
\hline 179 & 40 & & & $\mathrm{G} 2 \mathrm{~V}$ & 1 & \\
\hline 180 & 75 & $\mathrm{~B} 1 \mathrm{~V}$ & $\mathrm{a}$ & $\mathrm{B} 2 \mathrm{~V}$ & 1 & \\
\hline 182 & 86 & & & $\mathrm{~B} 4 \mathrm{~V}$ & 1 & \\
\hline 183 & 80 & $\mathrm{~B} 0.5 \mathrm{~V}, \mathrm{~B} 1 \mathrm{IV}, \mathrm{B} 0 \mathrm{~V}$ & $\mathrm{a}, \mathrm{b}, \mathrm{e}$ & B1III & 1,2 & \\
\hline 185 & 56 & $\mathrm{~B} 1 \mathrm{~V}, \mathrm{~B} 2 \mathrm{~V}$ & $a, b$ & $\mathrm{~B} 1.5 \mathrm{~V}$ & 1,2 & \\
\hline 188 & 78 & & & $\mathrm{~B} 2 \mathrm{~V}$ & 1 & \\
\hline 189 & 78 & & & $\mathrm{~B} 5 \mathrm{~V}$ & 1 & \\
\hline 191 & 75 & $\mathrm{~B} 2.5 \mathrm{~V}$ & $\mathrm{a}$ & $\mathrm{B} 2.5 \mathrm{~V}$ & 1 & \\
\hline 192 & 67 & $\mathrm{O} 5 \mathrm{~V}((\mathrm{f})), \mathrm{O} 5 \mathrm{~V}$ & $\mathrm{a}, \mathrm{b}$ & $\mathrm{O} 5 \mathrm{~V}((\mathrm{f}))$ & $1,2,3$ & \\
\hline 195 & 83 & & & G9V & 1 & \\
\hline 198 & 73 & & & G5III & 1 & \\
\hline 199 & 86 & & & G7Ib & 1 & \\
\hline 200 & 24 & & & $\mathrm{~B} 4 \mathrm{~V}$ & 1 & $\mathrm{~B}$ \\
\hline 208 & 69 & & & $\mathrm{~A} 1 \mathrm{~V}$ & 1 & \\
\hline 211 & 85 & $\mathrm{~B} 1 \mathrm{~V}, \mathrm{~B} 1 \mathrm{~V}$ & $a, b$ & $\mathrm{~B} 2 \mathrm{III}$ & 1,2 & \\
\hline 212 & 78 & $\mathrm{~B} 2 \mathrm{~V}$ & $\mathrm{a}$ & B3V & 1 & \\
\hline 213 & 72 & & & $\mathrm{~B} 5 \mathrm{~V}$ & 1 & B \\
\hline 214 & 63 & & & $\mathrm{~F} 2 \mathrm{~V}$ & 1 & \\
\hline 215 & 79 & & & K5II-III & 1 & \\
\hline 218 & 84 & & & B7V & 1 & \\
\hline 220 & 38 & & & F8V & 1 & \\
\hline 221 & 86 & B8II-III & $\mathrm{b}$ & B9.5V & 1 & \\
\hline 222 & 50 & & & G3V & 1 & B \\
\hline 224 & 58 & & & F0V & 1 & \\
\hline 225 & 87 & $\mathrm{~B} 2 \mathrm{~V}$ & $\mathrm{a}$ & $\mathrm{B} 2 \mathrm{~V}$ & 1 & \\
\hline 228 & 67 & & & F3V & 1 & \\
\hline 229 & 76 & & & $\mathrm{~B} 7 \mathrm{~V}$ & 1 & \\
\hline 232 & 81 & $\mathrm{O} 8 \mathrm{~V}((\mathrm{f})), \mathrm{O} 9 \mathrm{~V}$ & $a, b$ & $\mathrm{O} 7 \mathrm{~V}$ & $1,2,3$ & \\
\hline 236 & 75 & & & $\mathrm{~B} 8 \mathrm{~V}$ & 1 & \\
\hline 240 & 83 & & & $\mathrm{~B} 8 \mathrm{~V}$ & 1 & \\
\hline 241 & 29 & & & G5V & 1 & \\
\hline 244 & 75 & & & F0V & 1 & \\
\hline
\end{tabular}


Table 2. continued

\begin{tabular}{|c|c|c|c|c|c|c|}
\hline $\begin{array}{l}\text { star } \\
\text { No. }\end{array}$ & $\begin{array}{c}p \\
(\%)\end{array}$ & $\begin{array}{l}\text { spectral-type } \\
\text { published }\end{array}$ & sources & $\begin{array}{l}\text { spectral-type } \\
\text { new }\end{array}$ & $\begin{array}{l}\text { grating } \\
\text { No. }\end{array}$ & notes \\
\hline 248 & 74 & & & A1V & 1 & \\
\hline 250 & 77 & & & G8II & 1 & \\
\hline 252 & 85 & & & B3V & 1 & \\
\hline 255 & 84 & & & A5III & 1 & \\
\hline 258 & 86 & & & B8III & 1 & \\
\hline 259 & 81 & $\mathrm{~B} 1 \mathrm{~V}$ & $\mathrm{a}$ & $\mathrm{B} 1 \mathrm{~V}$ & 1 & \\
\hline 260 & 86 & $\mathrm{~B} 2 \mathrm{~V}, \mathrm{~B} 2 \mathrm{~V}$ & $a, b$ & B3IV & 1,2 & \\
\hline 262 & 85 & & & $\mathrm{~B} 2 \mathrm{~V}$ & 1 & \\
\hline 264 & 84 & & & $\mathrm{~B} 4 \mathrm{~V}$ & 1 & \\
\hline 266 & 86 & & & $\mathrm{~B} 5 \mathrm{~V}$ & 1 & \\
\hline 267 & 14 & & & $\mathrm{~A} 1 \mathrm{~V}$ & 1 & \\
\hline 276 & 83 & & & B3V & 1 & \\
\hline 277 & 85 & & & B3V & 1 & \\
\hline 278 & 83 & A0II-III & $\mathrm{b}$ & A5III & 1,2 & \\
\hline 279 & 46 & & & $\mathrm{~B} 7 \mathrm{~V}$ & 1 & \\
\hline 280 & 86 & & & A3III & 1 & \\
\hline 288 & 83 & $\mathrm{~B} 1 \mathrm{~V}, \mathrm{~B} 1.5 \mathrm{~V}$ & $\mathrm{a}, \mathrm{b}$ & B2III & 1,2 & \\
\hline 290 & 76 & F1V & $\mathrm{b}$ & F1III & 1,2 & \\
\hline 298 & 37 & & & $\mathrm{~A} 5 \mathrm{~V}$ & 1 & \\
\hline 300 & 25 & & & $\mathrm{~A} 3 \mathrm{~V}$ & 1 & B \\
\hline 301 & 76 & & & F5V & 1 & \\
\hline 303 & 44 & A0II-III & $\mathrm{b}$ & A0II-III & 1 & \\
\hline 308 & 64 & G5Ib-II & $\mathrm{b}$ & G5Ib-II & 1 & \\
\hline 310 & 84 & & & $\mathrm{~A} 3 \mathrm{~V}$ & 1 & \\
\hline 322 & 84 & & & A $5 \mathrm{~V}$ & 1 & B \\
\hline 330 & 85 & & & $\mathrm{~A} 7 \mathrm{~V}$ & 1 & B \\
\hline 332 & 85 & & & B3V & 1 & \\
\hline 339 & 77 & & & A $5 \mathrm{~V}$ & 1 & \\
\hline 346 & 81 & & & A3V & 1 & \\
\hline
\end{tabular}

Sources:

a: Massey et al. (1995).

b: Ishida (1970).

c: Hoag \& Applequist (1965).

d: Conti \& Alschuler (1971).

e: Hiltner (1956).

Notes:

A: showing $\mathrm{H} \alpha$ emission.

B: low $\mathrm{S} / \mathrm{N}$.

The spectra of a complete grid of MKK standards observed by us served as the basic frame for our spectral comparison and classification. Published atlases of grating spectra with similar resolution were also referenced. The main atlases used are those from Jacoby et al. (1984) and Turnshek et al. (1985).

Criteria of MKK classification in the classical region (3900 $\AA-4900 \AA$ ) are described and illustrated in many sources (Keenan \& McNeil 1976; Morgan et al 1978; Jaschek \& Jaschek 1987). These criteria were used in our spectral classification. As our spectra also covered the yellow-red region $(5000 \AA-8000 \AA)$, all the spectral features present in the whole wavelength range from blue to red could be examined and compared. Many authors have been engaged in seeking suitable criteria for MKK classification in non-classical regions (Turnshek et al. 1985; Silva \& Cornell 1992; TorresDodgen \& Weaver 1993; Danks \& Dennefeld 1994; Allen \& Strom 1995; Shi \& Hu 1999). As stated by these authors and illustrated by our spectra of observed MKK standards (Shi \& Hu 1999), a lot of spectral features (lines, blends or bands) show temperature or luminosity effect in the yellow-red region. Among these, the intensity ratio of blend $6495 / \mathrm{H} \alpha$ is a very good 
Table 3. Spectral classification of stars in NGC 654

\begin{tabular}{|c|c|c|c|c|c|c|}
\hline $\begin{array}{l}\text { star } \\
\text { No. }\end{array}$ & $\begin{array}{c}p \\
(\%)\end{array}$ & $\begin{array}{l}\text { spectral-type } \\
\text { published }\end{array}$ & sources & $\begin{array}{l}\text { spectral-type } \\
\text { new }\end{array}$ & $\begin{array}{l}\text { grating } \\
\text { No. }\end{array}$ & notes \\
\hline 5 & 95 & & & $\mathrm{~B} 8 \mathrm{~V}$ & 1 & \\
\hline 9 & 96 & & & $\mathrm{~B} 1.5 \mathrm{Ve}$ & $1,2,3$ & A \\
\hline 37 & 96 & & & K4III & 1 & \\
\hline 52 & 80 & & & B1.5Ie & 1,2 & $\mathrm{~A}$ \\
\hline 57 & 94 & & & B9III & 1,2 & \\
\hline 68 & 91 & $\mathrm{~A} 2 \mathrm{Ib}, \mathrm{A} 2 \mathrm{Ib}, \mathrm{A} 0 \mathrm{Ib}$ & $\mathrm{a}, \mathrm{b}, \mathrm{d}$ & A0Ib-II & $1,2,3$ & \\
\hline 71 & 96 & & & B1V & 1 & \\
\hline 72 & 93 & & & B3V & 1 & \\
\hline 76 & 95 & & & $\mathrm{~B} 1.5 \mathrm{~V}$ & 1 & B \\
\hline 78 & 94 & $\mathrm{~B} 1.5 \mathrm{IV}, \mathrm{B} 6 \mathrm{~V}$ & $\mathrm{a}, \mathrm{d}$ & B1.5III-IV & 1,2 & \\
\hline 79 & 94 & & & $\mathrm{~B} 2 \mathrm{~V}$ & 1 & \\
\hline 81 & 94 & & & B3V & 1 & \\
\hline 84 & 91 & & & $\mathrm{~B} 4 \mathrm{~V}$ & 1 & \\
\hline 85 & 93 & & & $\mathrm{~B} 2 \mathrm{~V}$ & 1 & \\
\hline 86 & 93 & & & $\mathrm{~B} 2 \mathrm{~V}$ & 1 & \\
\hline 88 & 92 & $\mathrm{~B} 1 \mathrm{~V}$ & $\mathrm{a}$ & $\mathrm{B} 1.5 \mathrm{~V}$ & 1,2 & \\
\hline 89 & 94 & & & $\mathrm{~B} 2.5 \mathrm{~V}$ & 1 & \\
\hline 90 & 94 & B2IV & $\mathrm{a}$ & $\mathrm{B} 1 \mathrm{~V}$ & 1 & \\
\hline 94 & 94 & & & $\mathrm{~B} 1 \mathrm{~V}$ & 1 & B \\
\hline 96 & 93 & & & $\mathrm{~B} 2 \mathrm{~V}$ & 1 & B \\
\hline 97 & 94 & & & $\mathrm{~B} 2 \mathrm{~V}$ & 1 & \\
\hline 98 & 94 & & & $\mathrm{~B} 1.5 \mathrm{~V}$ & 1 & \\
\hline 100 & 95 & & & $\mathrm{~B} 1 \mathrm{~V}$ & 1 & \\
\hline 101 & 36 & & & $\mathrm{~B} 1 \mathrm{~V}$ & 1 & \\
\hline 102 & 93 & & & $\mathrm{~B} 2 \mathrm{~V}$ & 1 & \\
\hline 103 & 95 & & & $\mathrm{~B} 2 \mathrm{~V}$ & 1 & \\
\hline 104 & 92 & & & $\mathrm{~B} 0 \mathrm{Ve}$ & 1,2 & A \\
\hline 106 & 96 & & & B3V & 1 & \\
\hline 108 & 96 & & & $\mathrm{~B} 2 \mathrm{~V}$ & 1 & \\
\hline 109 & 94 & B2II & $\mathrm{a}$ & B2II & 1,2 & \\
\hline 111 & 92 & F5Ia, F2Ia, F5Ia & $a, b, c$ & F5Ia & $1,2,3$ & \\
\hline 112 & 91 & & & $\mathrm{~B} 2.5 \mathrm{~V}$ & 1 & \\
\hline 113 & 93 & & & $\mathrm{~B} 1.5 \mathrm{~V}$ & 1 & \\
\hline 114 & 89 & $\mathrm{~B} 1 \mathrm{~V}, \mathrm{~B} 5 \mathrm{~V}$ & $\mathrm{a}, \mathrm{d}$ & $\mathrm{B} 1.5 \mathrm{~V}$ & 1,2 & \\
\hline 119 & 95 & & & $\mathrm{~B} 2 \mathrm{~V}$ & 1 & \\
\hline 124 & 95 & & & $\mathrm{~B} 1.5 \mathrm{~V}$ & 1 & \\
\hline 125 & 95 & $\mathrm{~B} 1.5 \mathrm{~V}, \mathrm{~B} 6 \mathrm{~V}$ & $\mathrm{a}, \mathrm{d}$ & $\mathrm{B} 1.5 \mathrm{~V}$ & 1 & \\
\hline 127 & 67 & & & B3V & 1 & B \\
\hline 130 & 90 & & & $\mathrm{~B} 1.5 \mathrm{~V}$ & 1 & \\
\hline 131 & 87 & B2IV & $\mathrm{a}$ & $\mathrm{B} 1.5 \mathrm{~V}$ & 1,2 & \\
\hline 133 & 85 & & & F3V & 1 & \\
\hline 135 & 96 & $\mathrm{~B} 1 \mathrm{~V}$ & $\mathrm{a}$ & $\mathrm{B} 1 \mathrm{Ve}$ & 1 & $\mathrm{~A}$ \\
\hline 147 & 64 & & & F8IV & 1 & \\
\hline 154 & 88 & & & $\mathrm{~B} 1.5 \mathrm{~V}$ & 1 & \\
\hline 161 & 63 & & & B9.5V & 1,2 & \\
\hline 170 & 88 & & & G8III & 1 & B \\
\hline
\end{tabular}

Sources:

a: Turnner (1976).

b: Sowell (1987).

c: Boulon et al. (1960).

d: Hoag \& Applequist (1965).

Notes:

A: showing $\mathrm{H} \alpha$ emission.

B: low $\mathrm{S} / \mathrm{N}$. 
Table 4. Spectral classification of stars in NGC 6823

\begin{tabular}{|c|c|c|c|c|c|c|}
\hline $\begin{array}{l}\text { star } \\
\text { No. }\end{array}$ & $\begin{array}{c}p \\
(\%)\end{array}$ & $\begin{array}{l}\text { spectral-type } \\
\text { published }\end{array}$ & sources & $\begin{array}{l}\text { spectral-type } \\
\text { new }\end{array}$ & $\begin{array}{l}\text { grating } \\
\text { No. }\end{array}$ & notes \\
\hline 1 & 79 & & & A0V & 1 & \\
\hline 3 & 0 & & & $\mathrm{G} 2 \mathrm{~V}$ & 2 & \\
\hline 4 & 91 & B0IVe & $\mathrm{a}$ & B0.5IV & 1,2 & \\
\hline 5 & 95 & & & $\mathrm{~B} 2 \mathrm{~V}$ & 1 & \\
\hline 8 & 97 & O9.5III & $\mathrm{a}$ & $\mathrm{O} 8 \mathrm{~V}$ & $1,2,3$ & \\
\hline 9 & 94 & & & $\mathrm{~A} 2 \mathrm{~V}$ & 1,2 & \\
\hline 13 & 67 & & & B7V & 1,2 & \\
\hline 14 & - & & & $\mathrm{B} 2 \mathrm{~V}$ & 1 & \\
\hline 17 & - & & & $\mathrm{B} 1.5 \mathrm{~V}$ & 1 & \\
\hline 18 & 97 & & & $\mathrm{~B} 2 \mathrm{~V}$ & 1,2 & \\
\hline 20 & 0 & & & G7V & 2 & \\
\hline 21 & 0 & & & $\mathrm{~K} 4 \mathrm{~V}$ & 2 & \\
\hline 24 & 94 & $\mathrm{~B} 1.5 \mathrm{~V}$ & $\mathrm{~b}$ & $\mathrm{~B} 1.5 \mathrm{~V}$ & 1,2 & \\
\hline 26 & 0 & & & A9V & 2 & \\
\hline 30 & 88 & & & $\mathrm{~B} 1.5 \mathrm{~V}$ & 1 & \\
\hline 32 & 95 & & & A0III & 1,2 & \\
\hline 33 & 0 & & & F5III & 2 & \\
\hline 36 & 96 & & & G8III & 1 & \\
\hline 42 & 0 & & & F3V & 2 & \\
\hline 46 & 85 & $\mathrm{~B} 0.5 \mathrm{Ib}, \mathrm{B} 0.5 \mathrm{Ia}$ & $\mathrm{b}, \mathrm{c}$ & $\mathrm{B} 0.5 \mathrm{Ib}$ & $1,2,3$ & \\
\hline 50 & 94 & & & B4V & 1 & \\
\hline 52 & - & & & $\mathrm{B} 4 \mathrm{~V}$ & 1 & A \\
\hline 53 & 0 & $\mathrm{G} 2 \mathrm{~V}$ & $\mathrm{~d}$ & G3V & 2 & \\
\hline 54 & 96 & $\mathrm{~B} 1 \mathrm{~V}, \mathrm{~B} 1.5 \mathrm{~V}, \mathrm{~B} 2 \mathrm{III}$ & $a, b, d$ & $\mathrm{~B} 1.5 \mathrm{~V}$ & 1,2 & \\
\hline 55 & - & & & G8V & 1 & \\
\hline 56 & 0 & & & G8V & 2 & \\
\hline 57 & 96 & $\mathrm{~B} 1 \mathrm{~V}, \mathrm{~B} 2 \mathrm{~V}, \mathrm{~B} 2 \mathrm{~V}$ & $a, b, d$ & $\mathrm{~B} 2 \mathrm{~V}$ & 1,2 & \\
\hline 59 & 91 & $\mathrm{~B} 2 \mathrm{~V}, \mathrm{~B} 5 \mathrm{~V}$ & $\mathrm{a}, \mathrm{d}$ & B3V & 1 & \\
\hline 60 & 0 & & & K4III & 2 & \\
\hline 61 & 0 & & & F1IV & 2 & \\
\hline 62 & 0 & & & K0III & 2 & \\
\hline 63 & - & & & G8II-III & 1 & \\
\hline 65 & - & & & G5V & 1 & \\
\hline 66 & - & & & B9V & 1 & \\
\hline 67 & - & & & F2III-IV & 1 & \\
\hline 68 & 94 & O9.5Ia, B0IV & $\mathrm{b}, \mathrm{c}$ & B1III & $1,2,3$ & \\
\hline 69 & 96 & $\mathrm{~B} 2 \mathrm{~V}$ & $\mathrm{a}$ & B3V & 1 & \\
\hline 71 & - & & & $\mathrm{B} 6 \mathrm{~V}$ & 1 & \\
\hline 72 & 95 & O7V((f)), O9.5Ia & $a, b$ & O9.5III & $1,2,3$ & \\
\hline 74 & 95 & $\mathrm{~B} 1.5 \mathrm{~V}, \mathrm{~B} 0.5 \mathrm{~V}$ & $a, b$ & B1V & 1,2 & \\
\hline 77 & 95 & B1III, B1III & $\mathrm{b}, \mathrm{c}$ & B1III & 1,2 & \\
\hline 78 & 92 & $\mathrm{~K} 8 \mathrm{~V}$ & $\mathrm{a}$ & $\mathrm{K} 4 \mathrm{~V}$ & 1,2 & \\
\hline 79 & - & & & F9V & 1 & \\
\hline 80 & 96 & & & $\mathrm{~B} 2.5 \mathrm{~V}$ & 1 & \\
\hline 81 & 97 & $\mathrm{~B} 1.5 \mathrm{~V}, \mathrm{~B} 0.5 \mathrm{~V}$ & $\mathrm{a}$ & $\mathrm{B} 1 \mathrm{~V}$ & 1,2 & \\
\hline 82 & - & & & F0V & 1 & \\
\hline 83 & 96 & O7V $((f))$ & $\mathrm{b}$ & O7V((f)) & $1,2,3$ & \\
\hline 84 & 96 & B0.5III, B0.5V & $b, d$ & $\mathrm{~B} 1 \mathrm{~V}$ & 1,2 & \\
\hline 85 & - & & & $\mathrm{B} 4 \mathrm{~V}$ & 1,2 & \\
\hline 86 & 95 & $\mathrm{O} 9 \mathrm{~V}, \mathrm{~B} 0.5 \mathrm{~V}$ & $a, b$ & $\mathrm{~B} 0.5 \mathrm{~V}$ & 1,2 & \\
\hline 87 & 0 & & & F8III & 2 & \\
\hline 88 & 96 & B0:Vpe, B1.5V & $a, b$ & $\mathrm{~B} 1 \mathrm{~V}$ & 1,2 & \\
\hline 89 & 76 & & & F5V & 1 & \\
\hline 92 & 67 & & & F8V & 1 & \\
\hline 93 & 93 & $09.5 \mathrm{~V}, \mathrm{~B} 1 \mathrm{~V}$ & $a, b$ & BOV & 1 & \\
\hline 94 & - & & & B8III & 2 & \\
\hline 95 & 0 & G0V: & $\mathrm{a}$ & F7V & 2 & \\
\hline 96 & 67 & & & F2IV & 1,2 & \\
\hline 97 & - & & & K3III & 1 & \\
\hline 98 & 0 & & & G2III & 2 & \\
\hline
\end{tabular}

luminosity indicator for late-type stars, with the intensity of the blend significantly enhanced in supergiants. These new criteria did help in our stellar classification, especially in cases when good $\mathrm{S} / \mathrm{N}$ could only be gained in the yellowred wavelength range.
When classifying a stellar spectrum, we first figured out an approximate temperature type (e.g., early-B, mid-B or late-B). The second step was to determine its luminosity class. After that a more accurate temperature type was obtained. The last two steps were often performed several times before a final accurate MKK type 
Table 4. continued

\begin{tabular}{rclllll}
\hline \hline star & $\begin{array}{c}p \\
\text { No. }\end{array}(\%)$ & $\begin{array}{l}\text { spectral-type } \\
\text { published }\end{array}$ & sources & spectral-type & grating & notes \\
new & & No. & \\
\hline 99 & - & & & B2V & 1 & \\
101 & 45 & & & A0V & 1 & \\
102 & - & & & F4V & 1 & A \\
103 & 92 & & G8III & 1 & \\
104 & 96 & B0.5V, B1V & a, b & B2III & 1,2 & \\
105 & 0 & & & K5V & 2 & \\
110 & 75 & F8Ib & a & F8Ib & $1,2,3$ & \\
113 & 75 & & & A7III-IV & 1 & \\
115 & 96 & B1V, B1.5III & a, b & B1.5III & 1,2 & \\
116 & - & & & B3V & 1 & \\
119 & 93 & B1V, B2.5V & a, b & B1V & 1,2 & \\
124 & - & & & G8III & 1 & A \\
126 & 0 & & & F3V & 2 & \\
135 & - & & & B2.5V & 1 & \\
136 & 0 & & & M6III & 2 & \\
141 & 0 & & & K2V & 1 & \\
143 & 81 & & & A2V & 1 & \\
\hline \hline
\end{tabular}

\author{
Sources: \\ a: Turner (1979). \\ b: Massey et al. (1995). \\ c: Hiltner (1956). \\ d: Hoag \& Applequist (1965).
}

Notes:

A: low $\mathrm{S} / \mathrm{N}$.

Table 5. Statistics of Be and B-type stars in the observed clusters

\begin{tabular}{lccc}
\hline \hline cluster & $\begin{array}{c}\text { number } \\
\text { of B-type stars }\end{array}$ & $\begin{array}{c}\text { number } \\
\text { of Be stars }\end{array}$ & $\begin{array}{c}\text { frequency } \\
\text { of Be stars }(\%)\end{array}$ \\
\hline IC 1805 & 33 & 1 & 3 \\
NGC 654 & 30 & 3 & 10 \\
NGC 6823 & 22 & 2 & 9 \\
Total & 85 & 6 & 7 \\
\hline \hline
\end{tabular}

could be fixed. When a program spectrum and a standard spectrum were compared, the whole spectrum as well as individual features corresponding to classification criteria were carefully examined. The program spectra were also compared with each other, to check that nothing went wrong during the process of spectral classification.

The errors in the MKK types classified by us have been estimated by: 1) comparing classification results of the two authors of this paper; 2) comparing results derived from spectra of different gratings (when existing); 3) comparing our results with those published in the literature for some brighter cluster stars; and 4) reclassifying the observed MKK standards; The errors of the resulting MKK types generally are smaller than 1 subclass in temperature types and about 1 subclass in luminosity classes. It should be pointed out that the luminosity class could be more easily determined using the spectra obtained with the grating of 600 lines/mm than the grating of 300 lines/mm. But even in the spectra of the lower dispersion luminosity classes I, III and V distinguished themselves from each other clearly.

\subsection{Results}

\subsubsection{IC 1805}

IC 1805 is the central cluster of the association Cas OB6. A proper motion survey was made by Vasilevskis et al. (1965) for 354 stars located in an area about two-thirds of a square degree and centered on $\mathrm{BD}+60^{\circ} 502$ (HD 15558) which is the brightest star of IC 1805. Spectra of 140 stars from this survey list were obtained by us. Of these observed stars, 126 have proper motion membership probabilities $p \geq 50 \%$ according to Zhao et al. (1985). Reduced spectra of all the program stars in IC 1805 are shown in Fig. ?? and Fig. ??, for 600 lines/mm and 300 lines $/ \mathrm{mm}$ grating respectively.

The results of the spectral classification on stars in IC 1805 are shown in Table 2. The star numbers from Vasilevskis et al. (1965) are listed in Col. 1. Proper motion membership probabilities from Zhao et al. (1985) are given in Col. 2. Spectral types published previously and their 
sources are presented in Cols. 3 and 4. MK types classified in this work are given in Col. 5. The grating(s) used in obtaining the spectra are listed in Col. 6 , with " 1 " for the 300 lines/mm grating, "2" for 600 lines/mm and " 3 " for 1200 lines $/ \mathrm{mm}$.

IC 1805 contains a lot of massive stars, about forty from O4.5 through B2. These are most probable cluster members, judging from both their proper motion membership probabilities and early spectral types. The presence of very early $\mathrm{O}$ type stars indicates a very young cluster age and makes this cluster an desirable object for the study of the star formation process as well as for the determination of IMF at higher masses.

\subsubsection{NGC 654}

NGC 654 is a young cluster also located in the Cassiopeia region as IC 1805. Stone (1977) conducted a proper motion study for 186 stars in the cluster area. Spectra of 46 stars from this study were taken by us. This sample of observed stars corresponding to all the stars with membership probabilities $p \geq 50 \%$ (Zhao et al. 1985) and brighter than $V=14$ m. Only one exception is star No. 101 with $p=36 \%$. Reduced spectra of all the program stars in NGC 654 are shown in Fig. ?? and Fig. ??.

The results of the spectral classification on stars in NGC 654 are shown in Table 3. The star numbers from Stone (1977) are listed in Col. 1. Proper motion membership probabilities from Zhao et al. (1985) are given in Col. 2. The other columns in Table 3 are similar to those in Table 2.

The earliest spectral type of around B0 means that NGC 654 is a more evolved young cluster than IC 1805. The presence of a few luminous stars, i.e., No. 111 (HD 10494, F5Ia), No. 68 (BD+61 315 , A0Ib-II), No. 2 (B1.5Ie) and No. 109 (B2II) makes NGC 654 an interesting cluster for the study of the evolution of massive stars.

\subsubsection{NGC 6823}

NGC 6823 is the central cluster of the association Vul OB1. Erickson (1971) made a proper motion survey of a region about $30^{\prime} \times 30^{\prime}$ centred on the central Trapezium system of NGC 6823. 146 stars were listed in that survey, but membership probabilities were computed only for the 92 stars brighter than $V=13^{\mathrm{m}} 0$ whose proper motions were better determined (Erickson 1971; Zhao et al. 1985). Spectra of 77 Erickson's stars were obtained by us. The observed stars went into three catagories: 1) 40 proper motion members, including all the stars with probability $p \geq 50 \%$ except for star No. 73 which is too close to a bright star; 2) 19 proper motion non-members; and 3) 18 stars fainter than $V=13 .^{\mathrm{m}} 0$ to which no proper motion membership probabilities were assigned. Reduced spectra of all the program stars in NGC 6823 are shown in Fig. ?? and Fig. ??.

The results of the spectral classification on stars in NGC 6823 are shown in Table 4. The star numbers from Erickson (1971) are listed in Col. 1. Proper motion membership probabilities from Zhao et al. (1985) are given in Col. 2. The other columns are similar to those in Table 2.

The earliest spectral type is O7, which suggests that the age of NGC 6823 may come between those of IC 1805 and NGC 654. The MKK types of two massive stars, No. 8 and No. 72, show some discrepancies among ours and other authors'. No. 8 is an O8V star here whereas it was classified as O9.5III by Turner (1979). No. 72 was assigned O7V((f)), O9Ia and O9.5III respectively by Turner (1979), Massey et al. (1995) and here. The spreads among spectral types for the above two stars(and maybe some others of similar cases in Table 2 through 4 ) as classified from time to time may indicate possible changes of spectral features due to some reason(s), rather than misclassification in the spectral types.

\section{Early type emission-line stars}

Quite a few stars showed at least $\mathrm{H} \alpha$ emission in our spectra. In IC 1805, the star No. 160 was confirmed as an Of star, and No. 113 an Be star (Ishida 1970; Massey et al. 1995). No. 70 is classified as an early type supergiant(B0.5Ia) with $\mathrm{H} \alpha$ emission. In NGC 654, four stars were found to have $\mathrm{H} \alpha$ emission. These include stars Nos. 9, 52, 104 and 135. No. 52 is classified as a supergiant, while the other three appear to be new Be stars from our spectral classification. In NGC 6823, The stars No. 4 and No. 88 were once classified as B0IVe and B0Vpe by Turner (1979). Both of them, however, did not show any distinct $\mathrm{H} \alpha$ emission in our spectra.

The frequency of Be stars has been discussed by many authors in the literature (Schild \& Romanishin 1976; Mermilliod 1982; Jaschek \& Jaschek 1983). Open clusters provide the desirable B-type parent population to which the Be stars can be compared. By definition, Be stars are non-supergiant B-type stars having at least once shown emission in one of the Balmer lines (Jaschek et al. 1981). Therefore, in IC 1805, NGC 6823 and NGC 654 respectively, one,two and three Be stars have been found as mentioned above. In Table 5, the number of all observed stars earlier than B2 (in Col. 2) and the number of Be stars ever found (in Col. 3) are given. All these Be stars also have types earlier than B2. According to both the proper motion surveys (mentioned in the previous section) and our spectral classification surveys over the three clusters, all of the cluster members earlier than B2 may well have been spectroscopically observed by us, on a statistical stand. The frequency of Be stars have been computed and given in Table 5. The averaged frequency of Be stars in these three clusters is $7 \%$. For the sake of comparison, we just 
list the results of similar work from two other sources below. A frequency of about $7 \%$ was also obtained by Schild \& Romanishin (1976) from a survey of 566 stars in 29 young open clusters, of which 41 Be stars were detected. By using the data of bright stars $(V \leq 6.5)$, Jaschek \& Jaschek (1983) derived a frequency of about $17 \%$ and $11 \%$ of Be stars among B0 through B2 stars and among all B-type stars respectively. It should be pointed out that a Be star may not exhibit emissions at the time the observation was carried out, and the percentage of Be stars among B-type stars at a given moment is smaller than that when all stars which were once Be are included (Jaschek \& Jaschek 1983). For a more reliable determination of the frequency of Be stars in open clusters, systematic detection of Be stars in more clusters should be made.

Detailed study on individual Be stars in open clusters such as those found in this work is of special interest, since the distances, ages and intrinsic colours can be inferred from the well-determined cluster parameters (Mermilliod 1982; Slettebak 1985). It will help in understanding the evolutionary status of Be stars and the origin of the envelops around them.

\section{Summary}

Three young open clusters with differential reddenings were selected as targets of a comprehensive spectral classification study. MKK types were obtained for 140, 46 and 77 stars in IC 1805, NGC 654 and NGC 6823 respectively. As these clusters are very young, most of the proper motion members are found to be early-type stars. Seven stars showed at least $\mathrm{H} \alpha$ emission in our spectra. Among these, three Be stars in NGC 654 were discovered by us. One Be and one Of star in IC 1805 were confirmed by our spectra. The other two were appropriately classified as early-type supergiants showing $\mathrm{H} \alpha$ emission.

The spectral classification work is just the first step to the systematical investigation of stellar contents and cluster properties (e.g., reddening, distance and age) of young open clusters and/or associations. Further study will be followed, based upon homogeneously combined data from spectroscopy, photometry, astrometry and so on.

Acknowledgements. The authors gratefully acknowledge Xinglong Observation-Station of the Joint Laboratories for Optical Astronomy for providing all the needed observing time. They also wish to express their sincere gratitude to Dr. Ram Sagar for his valuable comments on improving the paper. This research has made use of the BDA database, kindly offered by Dr. Jean-Claude Mermilliod, for bibliography information on MKK classification of stars in the clusters studied.

\section{References}

Allen L.E., Strom K.A., 1995, AJ 109, 1379

Boulon J., et al., 1960, Publ. Obs. Haute-Provence 4, No. 34

Conti P.S., Alschuler W.R., 1971, ApJ 170, 325

Danks A.C., Dennefeld M., 1994, PASP 106, 382

Erickson R.R., 1971, A\&A 10, 270

Hiltner W.A., 1956, ApJS 12, 215

Hoag A.A., Applequist L., 1965, ApJS 12, 215

Ishida K., 1970, Publ. Astr. Soc. Japan 22, 277

Jacoby G.H., Hunter D.A., Christian C.A., 1984, ApJS 56, 257

Jaschek C., Jaschek M., 1983, A\&A 117, 357

Jaschek C., Jaschek M., 1987, The claasification of Stars. Cambridge University Press, Cambridge

Jaschek M., Slettebak A., Jaschek C., 1981, Be Newslett. 4, 9

Joshi U.C., Sagar R., 1983a, J. R. Astr. Soc. Can. 77, 40

Joshi U.C., Sagar R., 1983b, MNRAS 202, 961

Keenan P.C., McNeil R.C., 1976, An atlas of spectra of the cooler stars. Ohio State University Press

Massey P., Johnson K.E., DeGioia-Eastwood K., 1995, ApJ 454,151

Mermilliod J.C., 1982, A\&A 109, 48

Mermilliod J.C., 1986, BIC DS 31, 175

Moffat A.F.J., 1972, A\&AS 7, 355

Morgan W.W., 1984, in: Garrison R.F. (ed.) The MK Process and Stellar Classification, p. 18

Morgan H.L., Keenan P.C., 1973, ARA\&A 11, 29

Morgan W.W., Abt H.A., Tapscott S.W., 1978, Revised MK spectral atlas for stars earlier than the sun. Yerkes Observatory and Kitt Peak National Observatory

Sagar R., 1987, MNRAS 228, 483

Sagar R., Joshi U.C., 1981, ApSS 75, 465

Sanders W.L., 1972, A\&A 16, 58

Schild R.E., Romanishin W., 1976, ApJ 204, 493

Shi H.M., Hu J.Y., 1999, Acta Astrophys. Sin. (in press)

Silva D.R., Cornell M.E., 1992, ApJS 81, 865

Slettebak A., 1985, ApJS 59, 769

Sowell J.R., 1987, ApJS 64, 241

Stone R.C., 1977, A\&A 54, 803

Stone R.C., 1980, PASP 92, 426

Torres-Dodgen A.V., Weaver W.B., 1995, PASP 105, 693

Turner D.G., 1976, AJ 81, 87

Turner D.G., 1979, J. R. Astro. Soc. Can. 73, 74

Turnshek D.E., Turnshek D.A., Craine E., Boeshaar P.C., 1985, An atlas of digital spectra of the cool stars. Tucson: Western Reasearch Co.

van den Ancker M.E., The P.S., Feinstein A., Vazquez R.A., der Winter D., Perez M.R., 1997, A\&AS 123, 63

Vasilevskis S., Sanders W.L., Van Altena F., 1965, AJ 70, 806

Zhao J., Tian K, Jing J., Yin M., 1985, Special Issue for Tables of Membership for 42 Open Clusters. Shanghai Astronomical Observatory, Chinese Academy of Sciences 

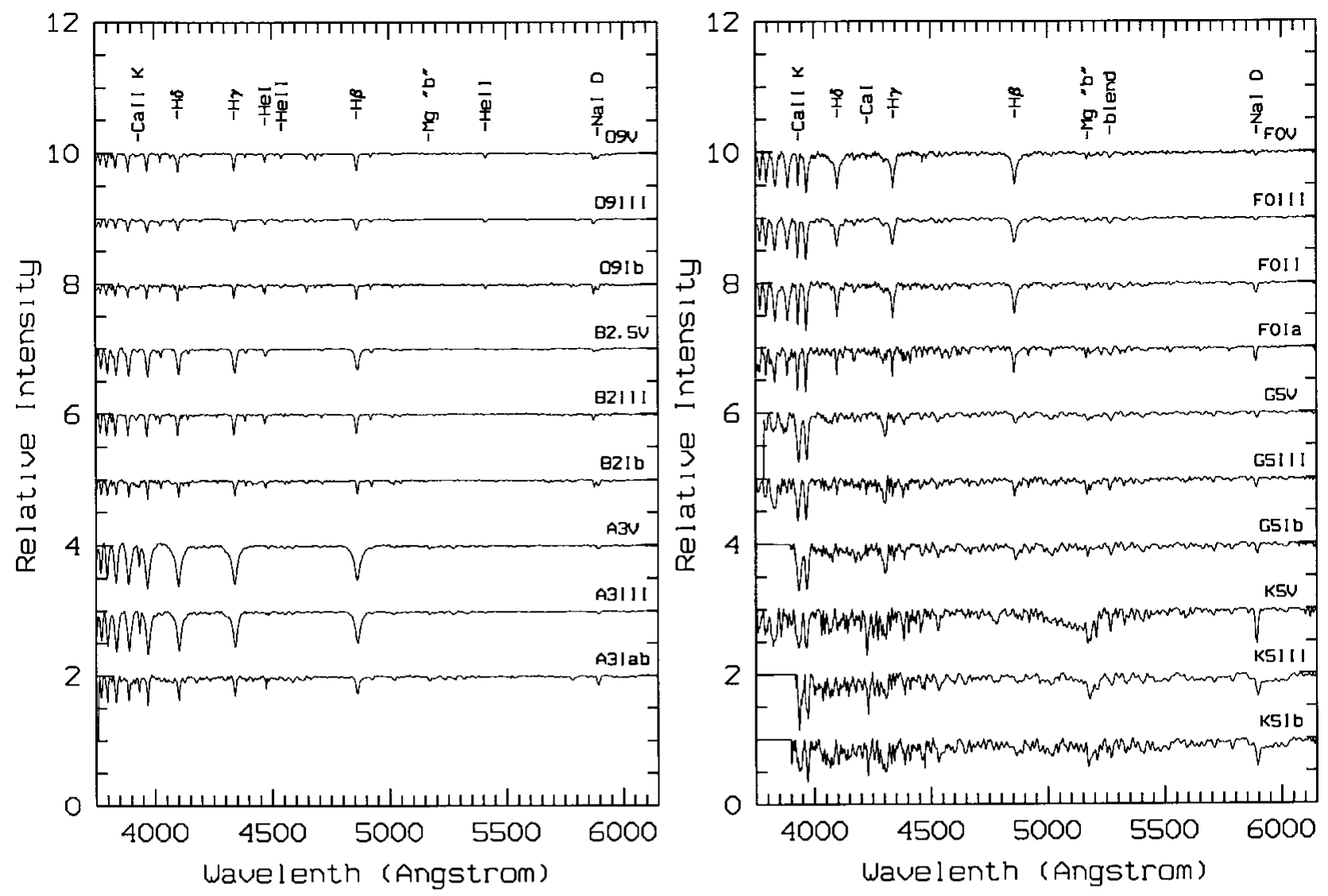

Fig. 1. Representative spectra of MK standards taken with 600 lines/mm grating
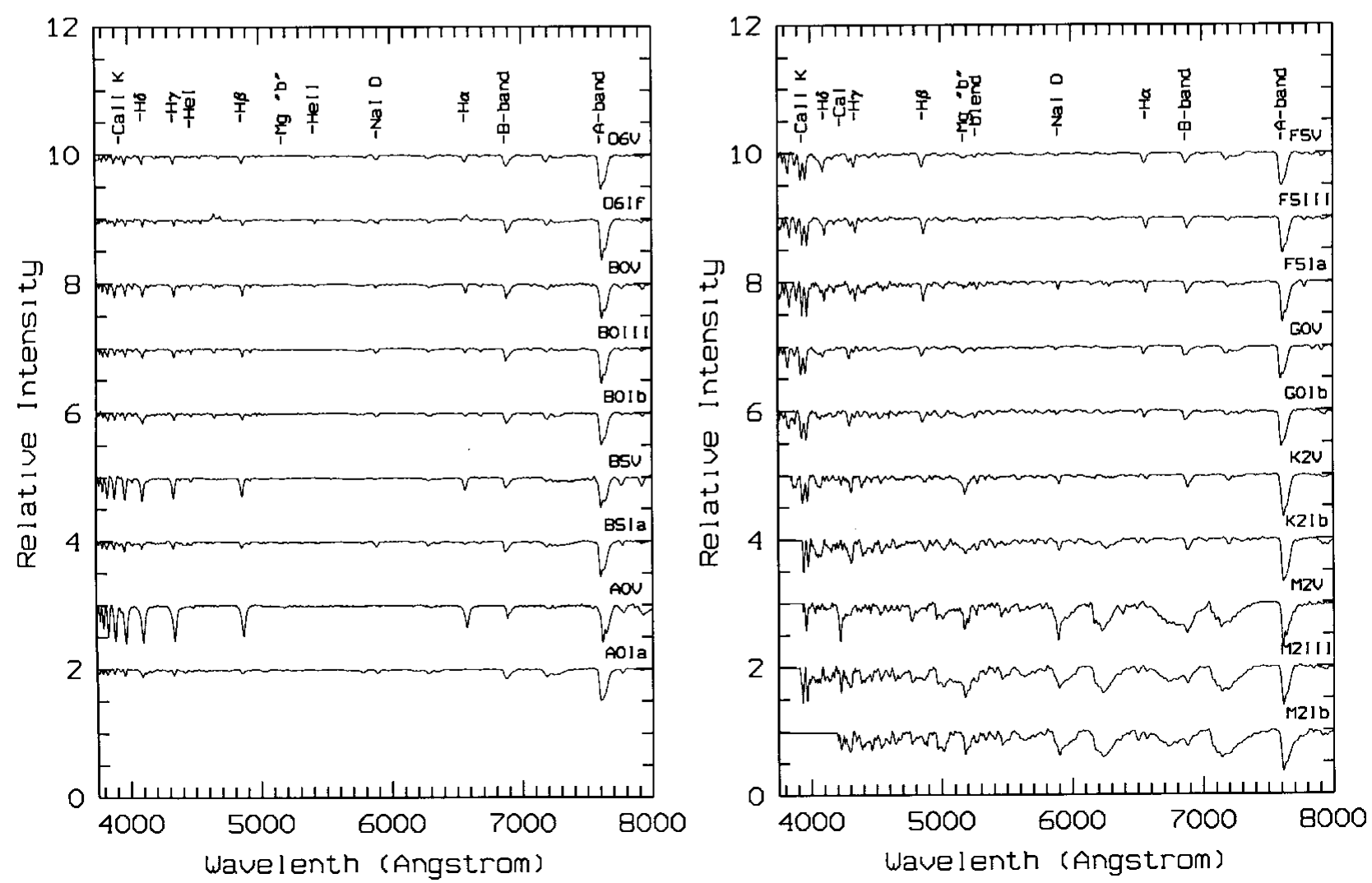

Fig. 2. Representative spectra of MK standards taken with 300 lines/mm grating 

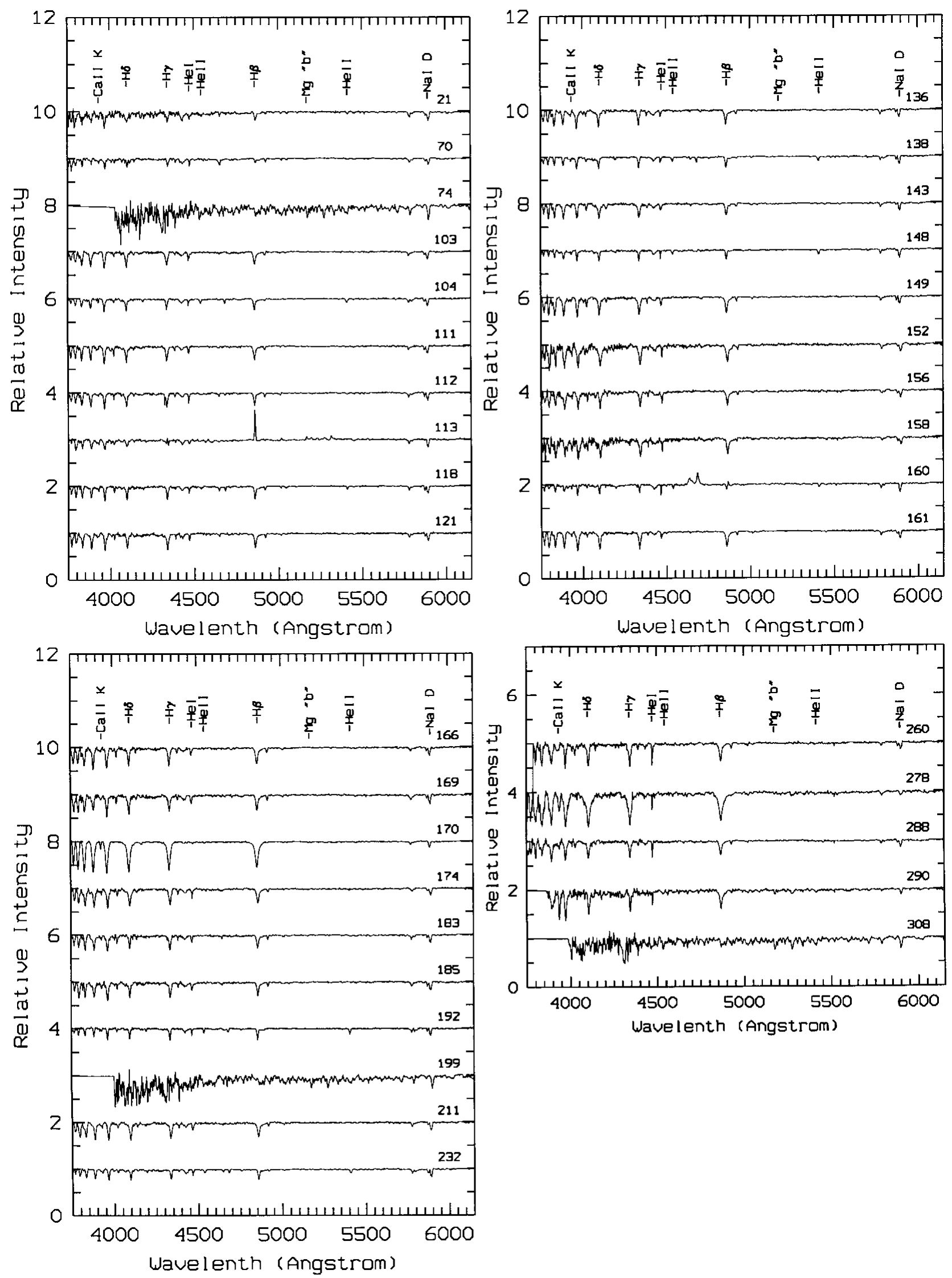

Fig. 3. Spectra of program stars in IC 1805 taken with 600 lines $/ \mathrm{mm}$ grating 

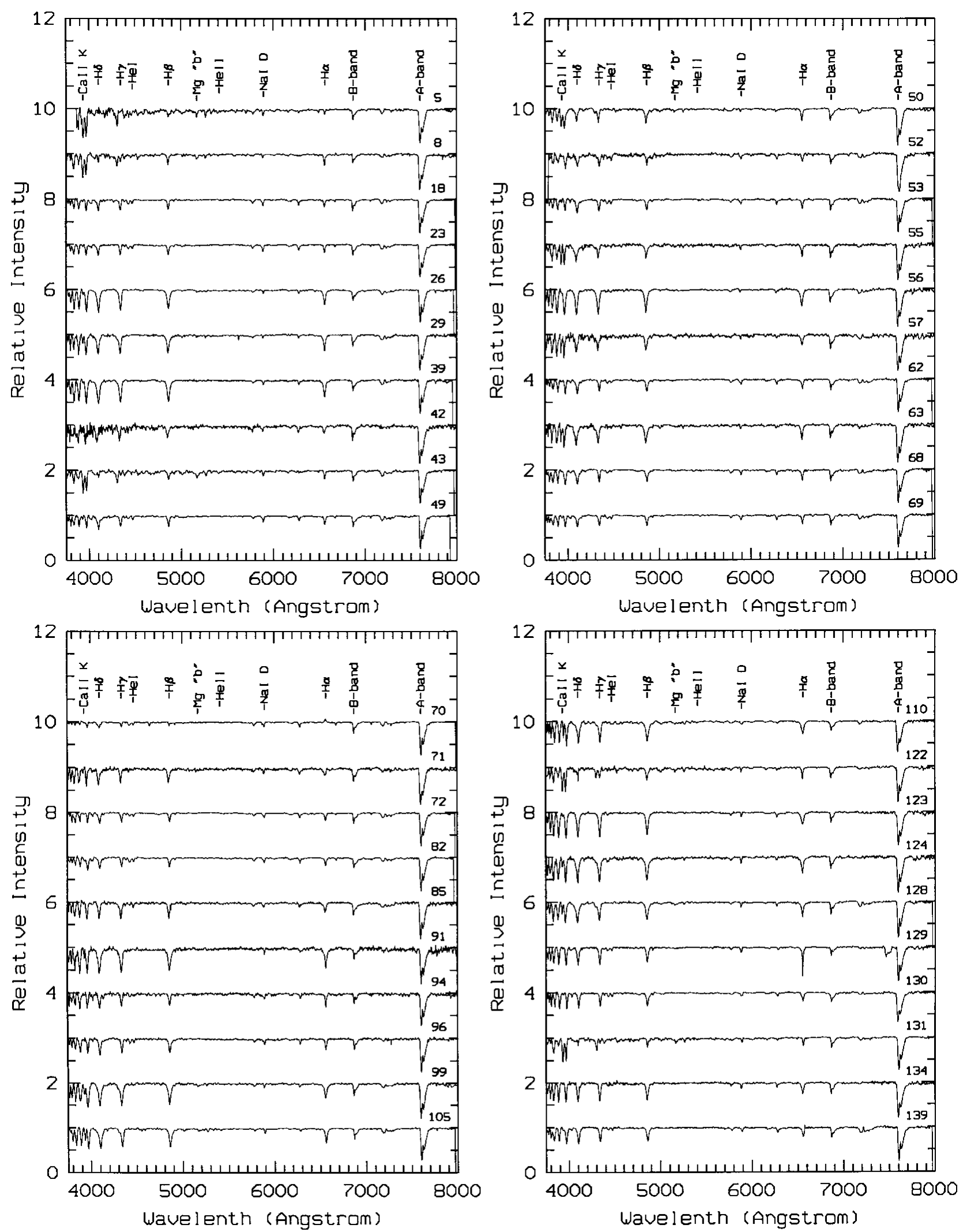

Fig. 4. Spectra of program stars in IC 1805 taken with 300 lines $/ \mathrm{mm}$ grating 

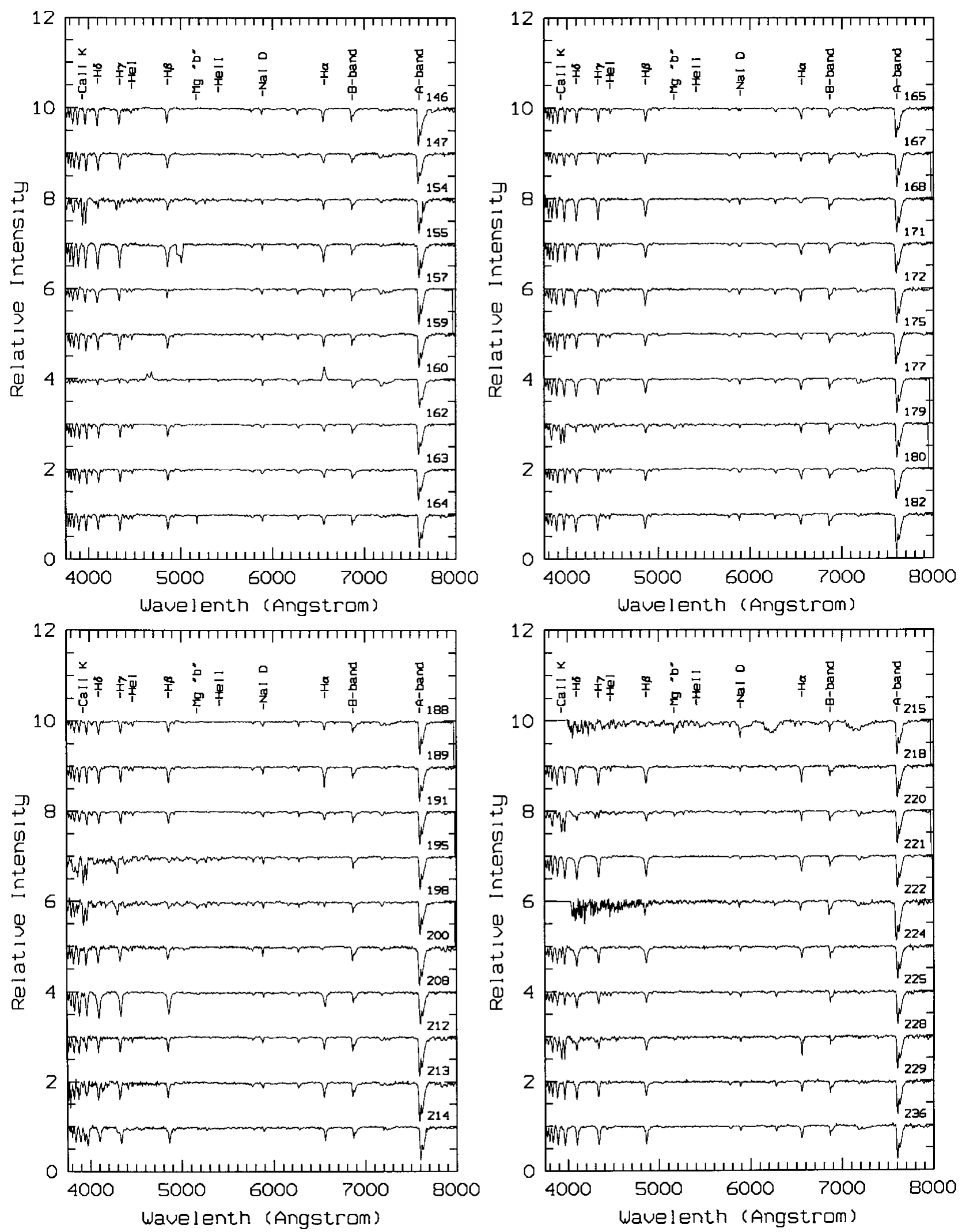

Fig. 4. continued 

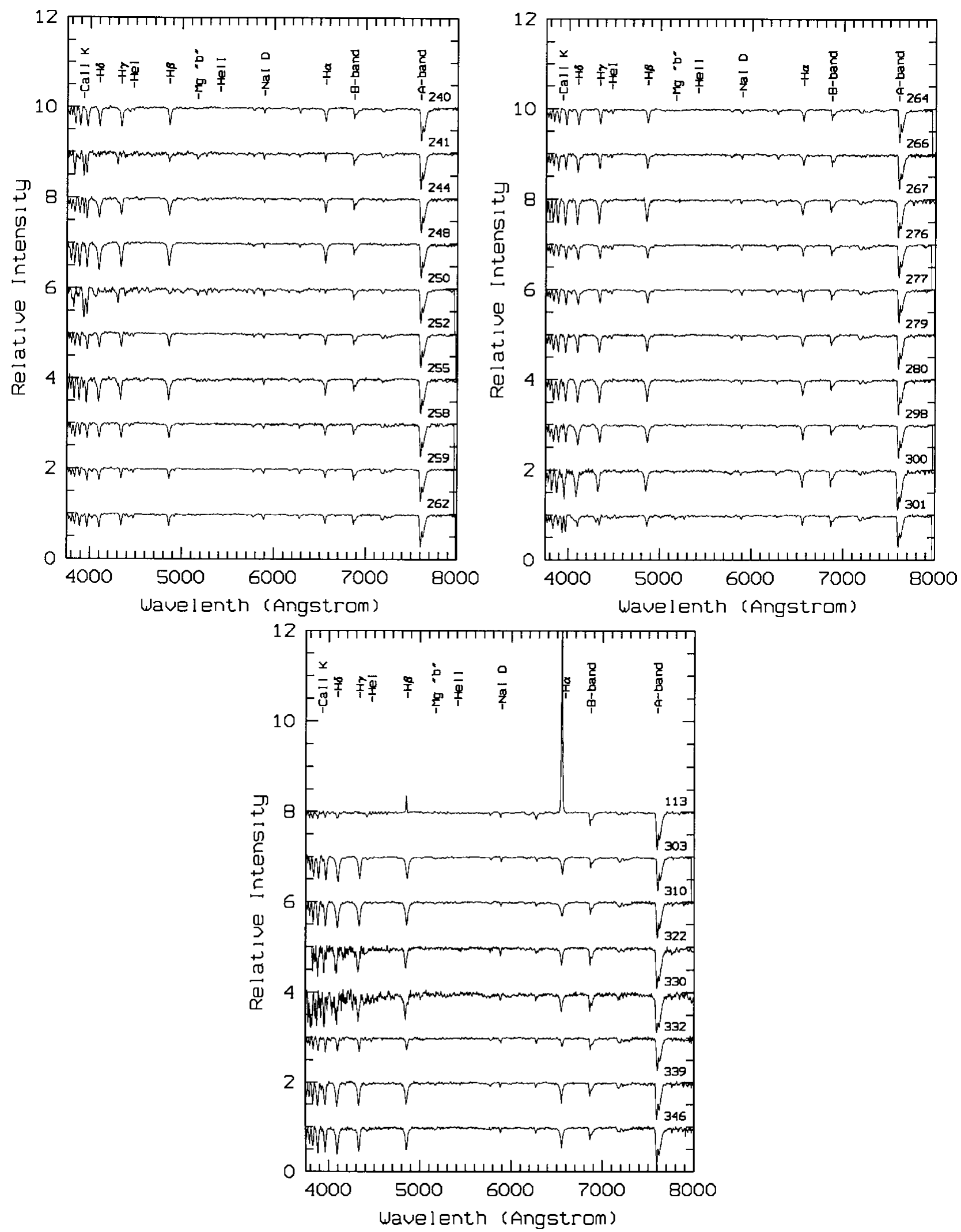

Fig. 4. continued 


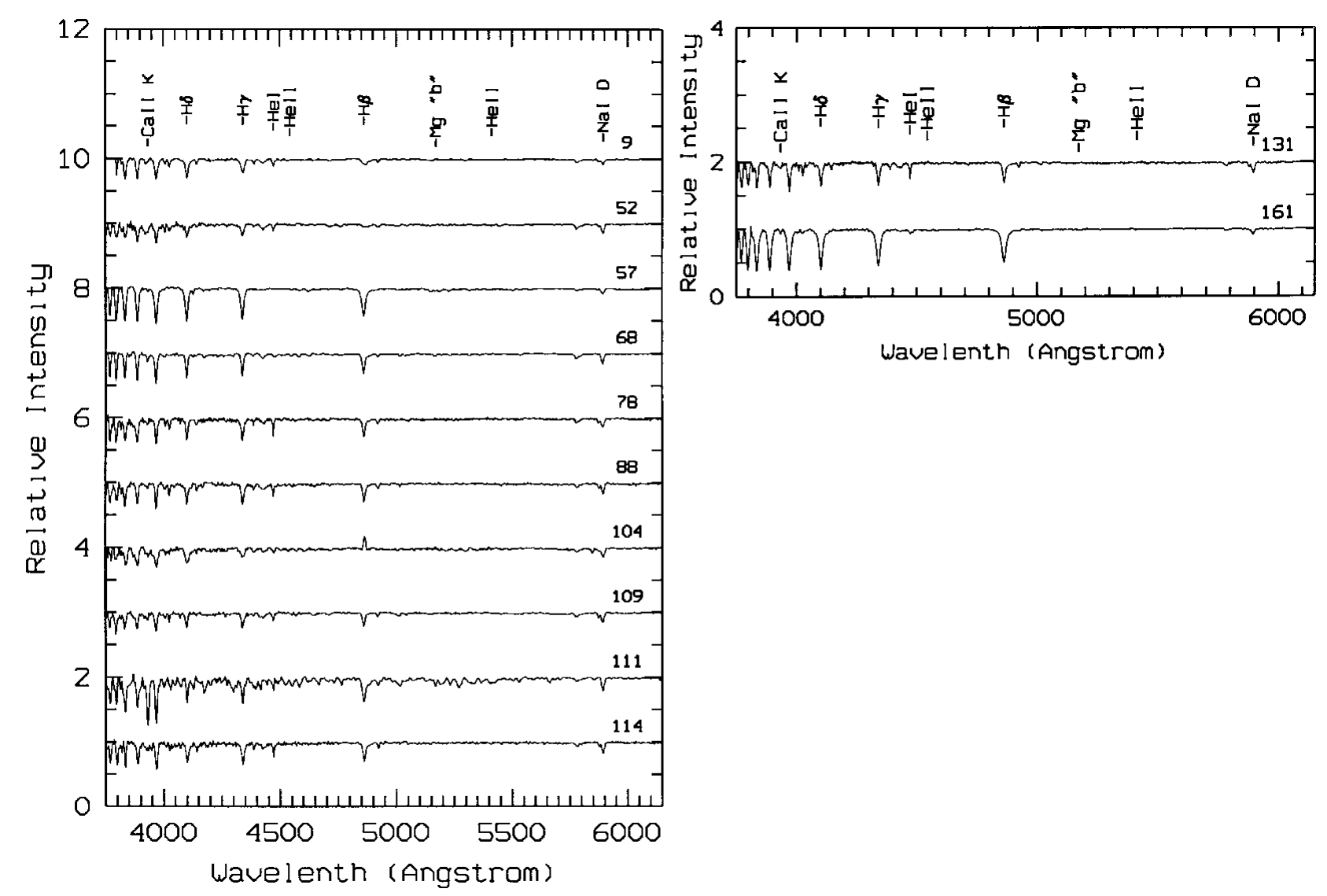

Fig. 5. Spectra of program stars in NGC 654 taken with 600 lines/mm grating
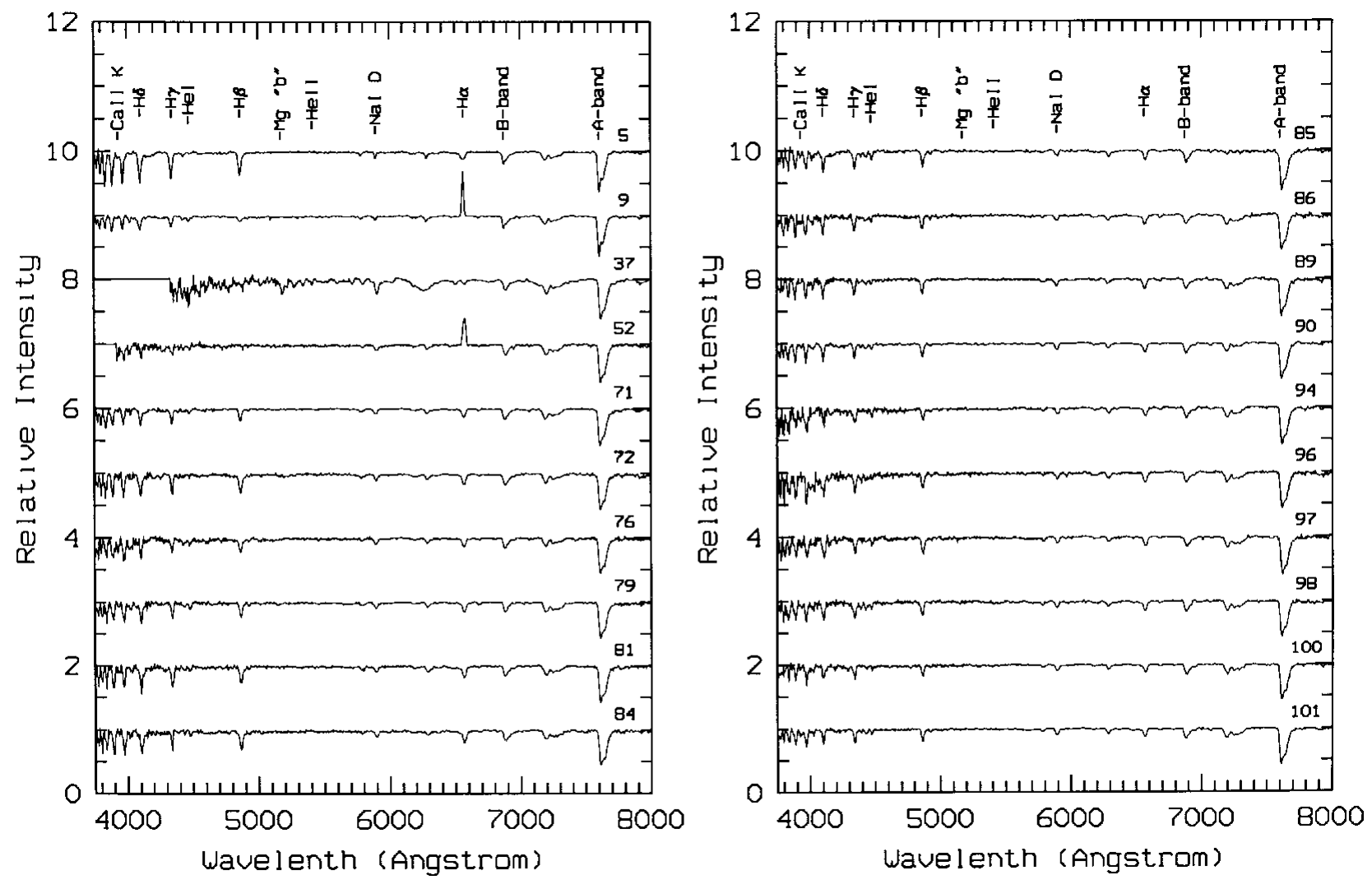

Fig. 6. Spectra of program stars in NGC 654 taken with 300 lines/mm grating 

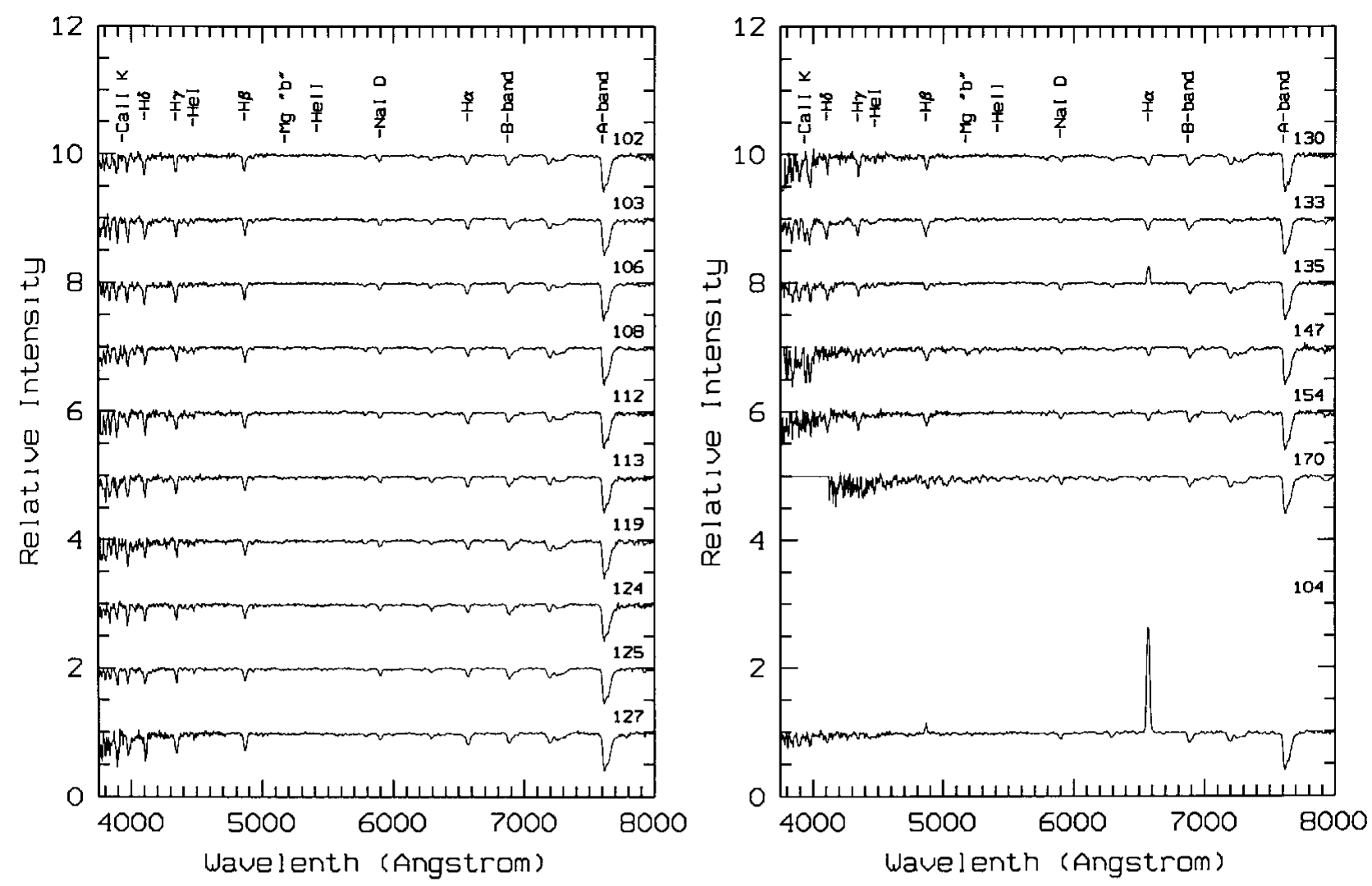

Fig. 6. continued
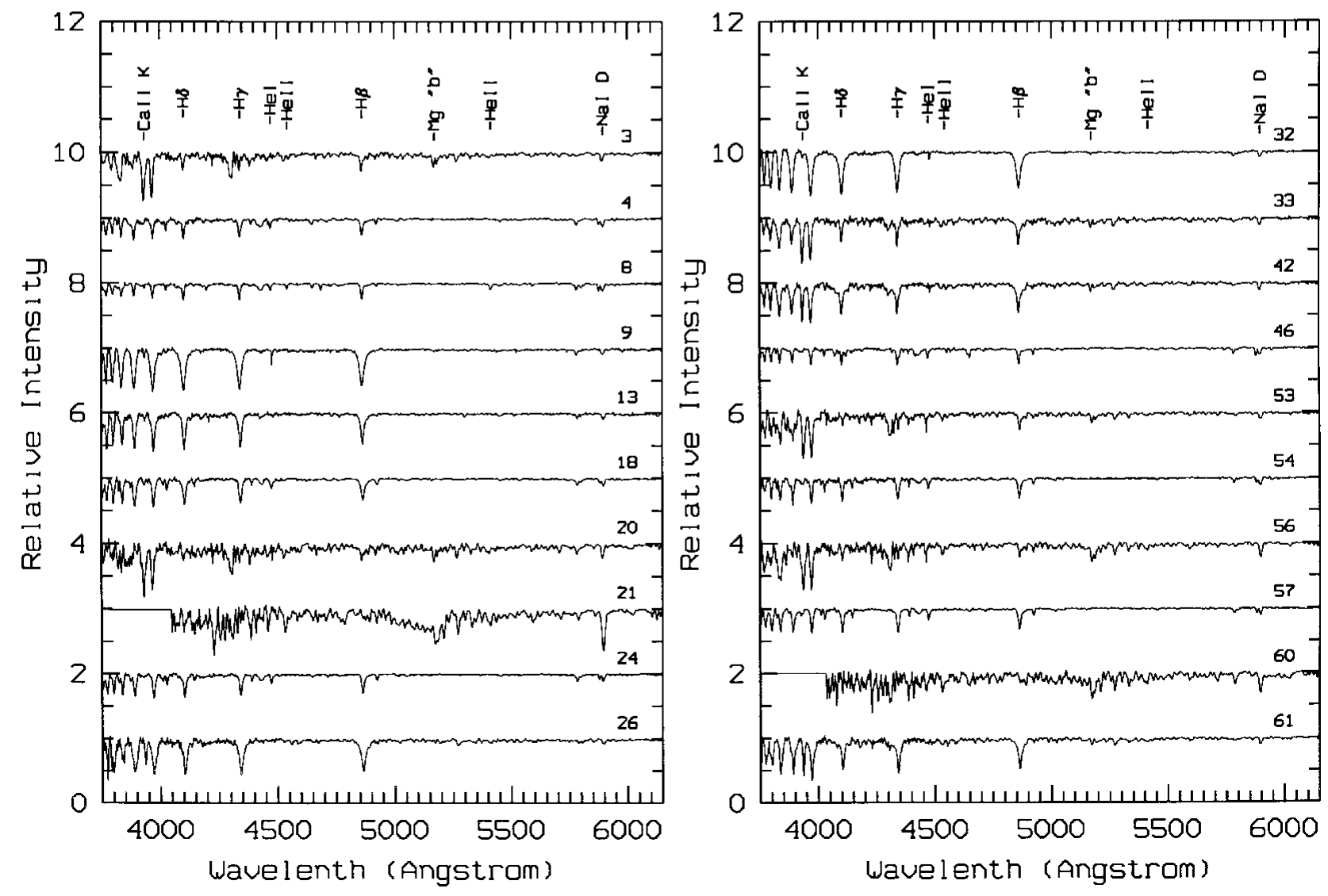

Fig. 7. Spectra of program stars in NGC 6823 taken with 600 lines $/ \mathrm{mm}$ grating 

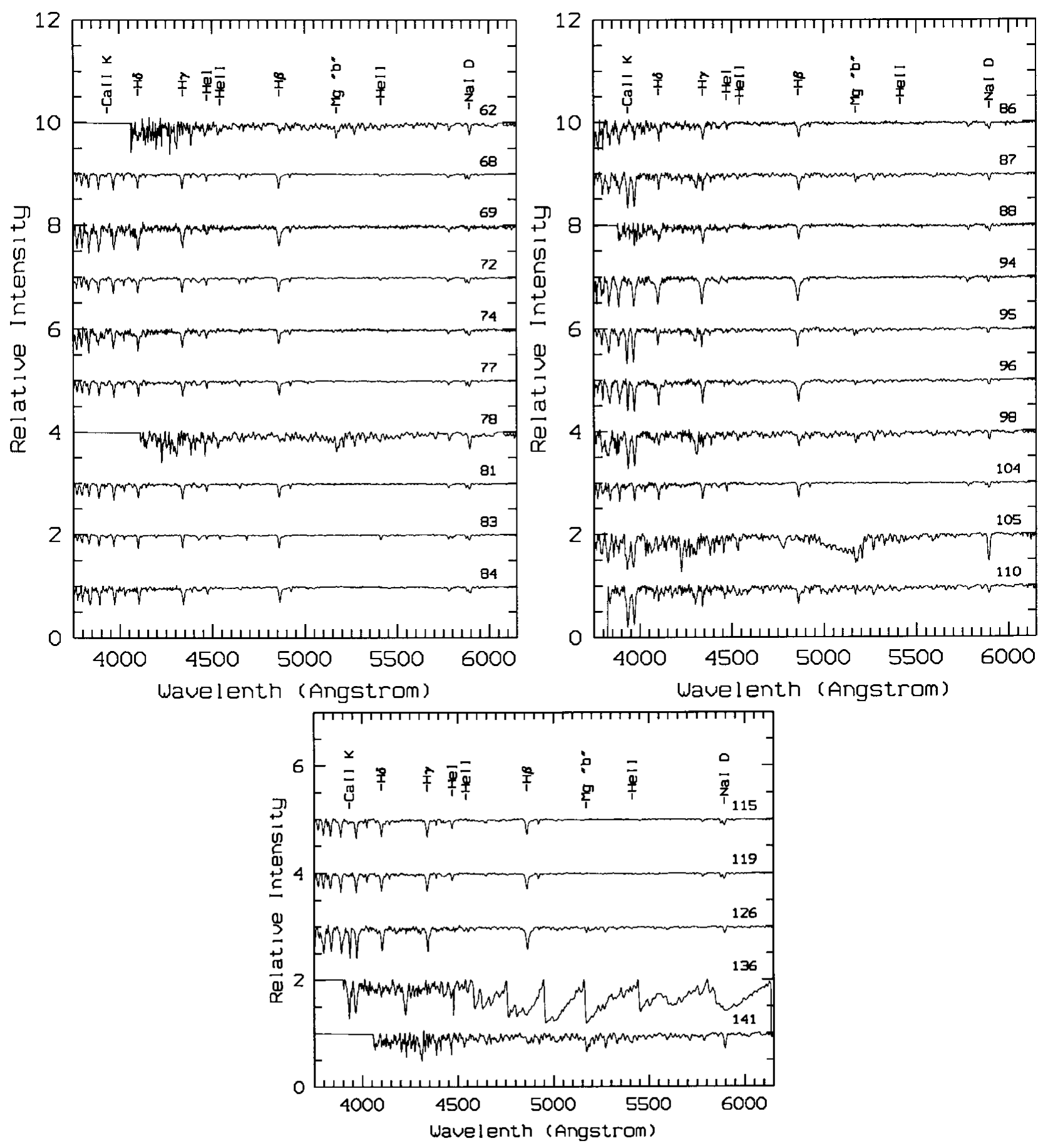

Fig. 7. continued 

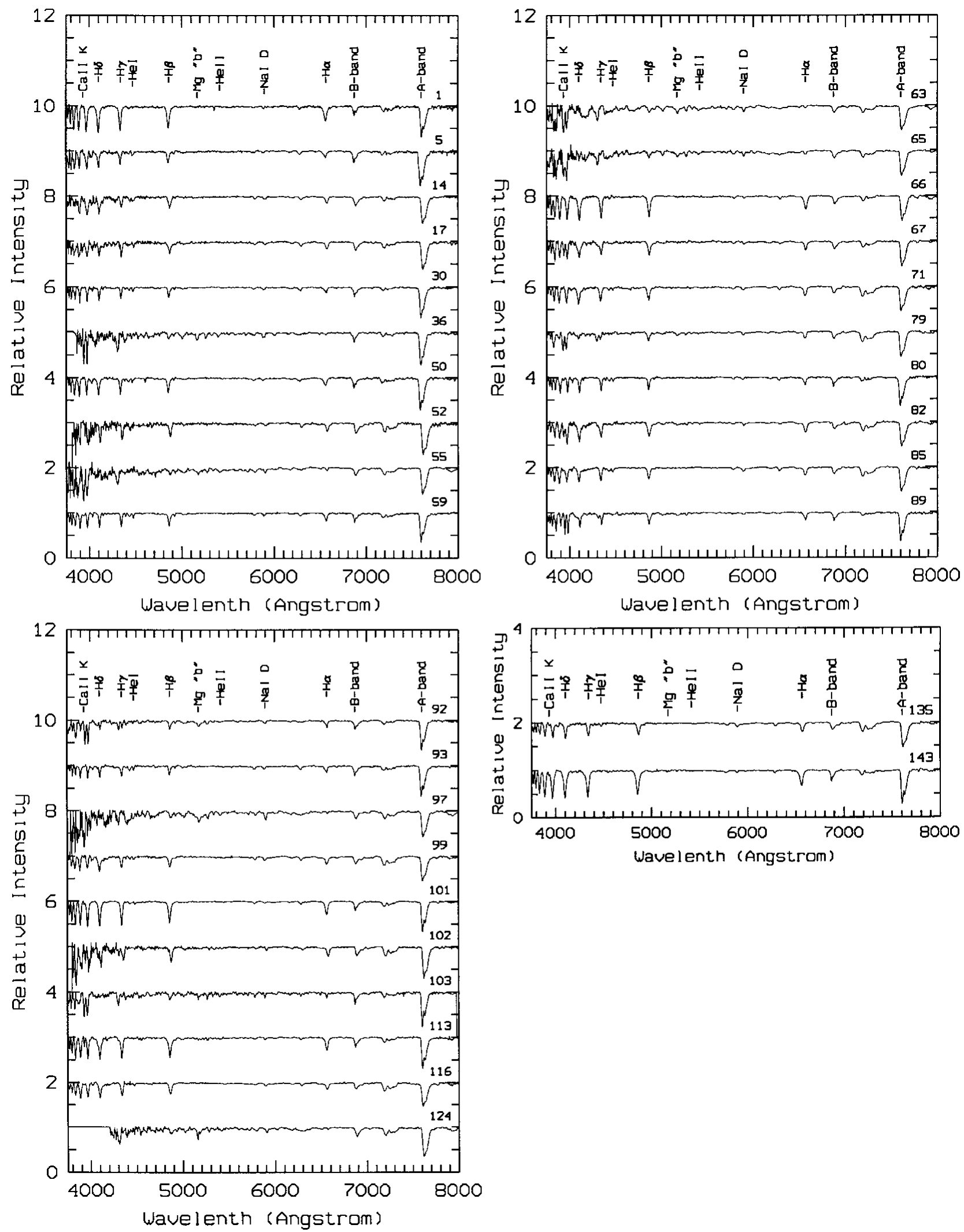

Fig. 8. Spectra of program stars in NGC 6823 taken with 300 lines/mm grating 